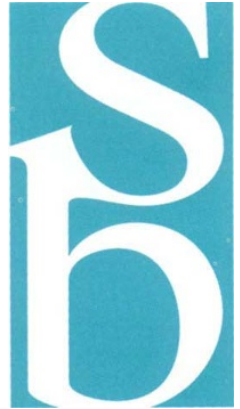

\section{A familiar ring}

While the publication of the model of the structure of DNA in 1953 immediately suggested a means by which the information it contained could be copied ${ }^{1}$ the detailed architecture of the copying machinery is only now being revealed. A further step on this pathway is provided by the structure of one of the accessory proteins of the eukaryotic replication complex, the yeast homologue of proliferating cell nuclear antigen (PCNA) reported in the latest issue of Cell.

Accessing and copying the data encoded in the genome is a mechanistically complex process: the double helix must be pried open, the strands held apart, the sequence of bases read and copied (once only), the two replication

\author{
IMAGE \\ UNAVAILABLE \\ FOR \\ COPYRIGHT \\ REASONS
}

Fig. 1 Protein with a hole. The electron density map (red) superimposed on the alpha carbon backbone (yellow) of the final refined model of the PCNA trimer. Mercury atoms (two per monomer) used in the structure determination are shown as blue spheres (reproduced from ref. 1 with permission; copyright Cell Press) forks translated along the DNA double helix, over-winding ahead of these regions alleviated, and so on. Copying presents a particular biophysical challenge in as much as it is done with great speed - rapid doubling times can be vital for survival (especially among prokaryotes) and genomes may be very large (some metazoans) - and with great accuracy. The rapidity with which the chromosomal replicase complexes can copy the genome (DNA polymerase III of Escherichia coli can copy 750 base pairs a second) is in large part conferred by a distinct non-catalytic subunit known as the processivity factor.

PCNA is the processivity factor of human DNA polymerase $\Delta$; the $\beta$-subunit of Pol III and the gene 45 protein are equivalent proteins in E. coli and T4 bacteriophage, respectively. Biochemical evidence had suggested that the interaction of these proteins with DNA is topological, rather than chemical, and this was dramatically confirmed by the determination of the structure of the $\beta$ subunit dimer ${ }^{3}$. The ring shape of the dimer suggested that the protein functions as a sliding clamp, wrapped around the DNA, both tethering the replication complex to the DNA and facilitating high processivity along the unravelling chromosome.

Surprisingly, although PCNA is known to function in an analogous manner to the E.coli $\beta$-subunit and the gene 45 protein, it has no significant sequence homology with either of these prokaryotic proteins. Nonetheless the shared function suggests the possibility of a similar structure. And this is indeed the case. The overall structure of the yeast PCNA, and the E. coli $\beta$-subunit, is that of a closed, sixpointed circular ring (Fig. 1). Despite the fact that PCNA is a trimer and the $\beta$-subunit is a dimer the two structures are remarkably similar. Each consists of six repeated domains (three per monomer in the $\beta$-subunit and two 
per monomer in PCNA) consisting of two antiparallel $B$-sheets closely apposed at one edge and splayed apart at the other by two $\alpha$-helices. The six domains in the three monomers (or two dimers for the $B$-subunit) come together to form six concave $B$-sheets on the outside of the ring. A long loop runs diagonally above and across the curved surface of several B-sheets, at right angles to the $B$-strands, evoking an image of bands holding the staves of a barrel together ${ }^{2}$. The inside of the rings is lined with twelve $\alpha$-helices.

What of the interaction with DNA? Although the average internal diameter of the PCNA ring is slightly smaller than that of the $ß$ subunit ( $34 \AA$ versus $38 \AA$ ) both can accommodate double helical B-form DNA, which has an average diameter of $18 \AA$. The twelve helices lining the central cavities of both PCNA and the $B$-subunit are oriented at right angles to the local direction of the grooves in the DNA passing through the ring, no doubt to minimize base-specific interaction and maintain a more general interaction with the phosphate backbone. Furthermore, both the $B$-subunit and PCNA have a net negative charge, yet there is a concentration of conserved, positively chargd residues lining the inside of the ring. This is a feature likely to facilitate sliding along the negatively charged, grooved surface of the $\mathrm{DNA}^{3}$.

The $\beta$-subunit clamp is attached to primed DNA by an ATP-driven clamploader complex and thence provides a platform from which the polymerase complex can interact with the unravelled DNA. Yet the very nature of DNA - the two antiparallel strands - also demands that the polymerase be able to hop on and off the lagging (5'-to-3') strand at a moments notice to generate short Okazaki fragments. How is this achieved? It would seem that when the fully active complex encounters a down-stream duplex (such as the 5 ' end of an Okazaki fragment) the Pol III assembly rapidly dissociates from the clamp, leaving it behind ${ }^{4,5}$. The polymerase then reassociates with another $B$-subunit and clamp- loader already attached to a new primed template. The prokaryotic (and presumably eukaryotic) sliding clamps thus provide a means by which the replication complex can rapidly switch templates, while at the same time maintaining high processivity on, and low rates of dissociation from, the leading DNA strand.

What of the stranded clamps? There are some suggestions that they may be used by other systems that interact with DNA such as the UV excision repair machinery and the cell cycle control apparatus. Certainly, it is known that PCNA interacts directly with the p21 cyclin dependent protein $\mathrm{ki}$ nase, which in turn is positively regulated by the p53 tumour suppressor protein ${ }^{6}$. Thus, PCNA provides a direct link between the cell cycle and DNA replication. The clamp and clamp-loading proteins of the T4 phage (genes 45, 62 and 44) have also been implicated in the transcriptional regulation of the T4 late genes ${ }^{7}$. The proteins act at an enhancer-like site and are suggested to 'track' (or slide) along the DNA until they encounter a promoter where, in combination with other T4-encoded transcription factors, they activate gene transcription. Given that DNA 'looping' is not necessarily excluded it is suggested that the clamp is not only delivering the transcription factor to its site of action but is also providing it in an active conformation. It will be interesting to see if these DNA clamps are used in yet other DNA-based systems.

1. Watson, J.D. \& Crick, F. Nature 171, 737-738 (1953). 2. Krishna, T.S.R., Kong, X-P., Gary, S., Burgers, P.M. \& Kuriyan, J. Cell in the press (1994).

3. Kong, X-P., Onrust, R. O'Donnell, M. \& Kuriyan, J. Cell 69, 425-437 (1992).

4. Stukenberg, P.T., Turner, J. \& O'Donnell Cell 78, 877-887 (1994). 5. Hacker, K. \& Alberts, B. J. biol. Chem. 269 in the press.

6. Waga, S., Hannon, G.J., Beach, D. \& Stillman, B. Nature $369,574-578$ (1994).

7. Herendeen, D.R., Kassavetis, G.A. \& Geiduschek, E.P. Science 256, 1298-1303 (1994).

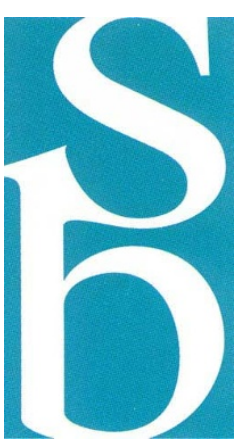

